\title{
Integration of Tungsten Micro-Heaters and Polymer Microfluidic for the Cell Sorting Application
}

\author{
Bivragh Majeed, Lut Van Acker, Koen De Wijs and Chengxun Liu \\ IMEC Leuven \\ IMEC, Kapeldreef 75, B3001 Leuven, Belgium. \\ Ph: +32-(0)16-28 7735; Fax: +32-(0)16-28 1097 \\ E-mail: bivragh.majeed@imec.be
}

\begin{abstract}
Cell sorting is an important diagnostic tool in medical field to separate different type of cells. Various techniques are employed for sorting with main mechanism being cell analysis and then separation either by electric or magnetic field. In this paper we explain the fabrication process for tungsten microheaters on silicon and polymer microfluidic for a formation of a generic platform that can be used for the various cell sorting applications. Polymer material is not only used for the formation of channel for fluidics movement but also used as bonding interface between the silicon wafer and the glass. We report on the bonding strength of the polymer material. It shows that our devices can withstand a pressure in excess of 3 bar which is more than sufficient for most microfluidic applications such as cell sorting. Characterization of micro stream bubble generation life cycle is presnted. The sorting efficiency of our device is reported, with initial characterization done beads to determine sorting rate and sorting yield. We achieved sorting rate of 1,000 beads/s with sorter purity of up to $98 \%$ in initial investigations.
\end{abstract}

\section{Introduction}

Detection and analysis of cells in a sample is very important tool for various medical diagnosis. Sorting of these cells can be broadly divided into two methods: bulk sorting and single cell sorting. Bulk sorting refers to collections of target cells in one step, while single sorting refers to analyzing individual cells. Various methods for bulk sorting include, centrifugation, cell culture and filtration, while one of the main method for sing cell sorting is flow cytometry [1]. Flow cytometry is a powerful tool that can provide quick information about a population of cells in a short time. Majority of cell sorting techniques are based on Fluorescence activated cell sorting (FACS). It which allows for separation of different type of cells at a very high throughput $[2,3]$. The cell travel along a channel illuminated by laser and main principle behind the separation is deflection a charge cell droplets by electric field. There has been many studies related to this field $[2,3]$ and even commercial equipment are available for FACS but they are bulky, expensive and not available at point of initial diagnosis [4]. At imec, we have been working on developing a generic platform that combines the benefits of CMOS compatible process with the flexibility of polymer microfluidics $[5,6,7]$. The targeted device will be low cost, disposable, require small sample volume and available at point of care.

The basic principle of our device is generation of a jet flow, triggered at a certain time, allowing for cell to be pushed in a certain direction. The process start with detection of incoming cell, once the target cell is identified, heat pulse on a heater element is applied. This will create a bubble jet flow The steam bubbles have a minimum life time of less than $10 \mu \mathrm{s}$. These micro bubble disturb the flow and thus enable very controlled sorting. The process is schematically shown in Figure 1.

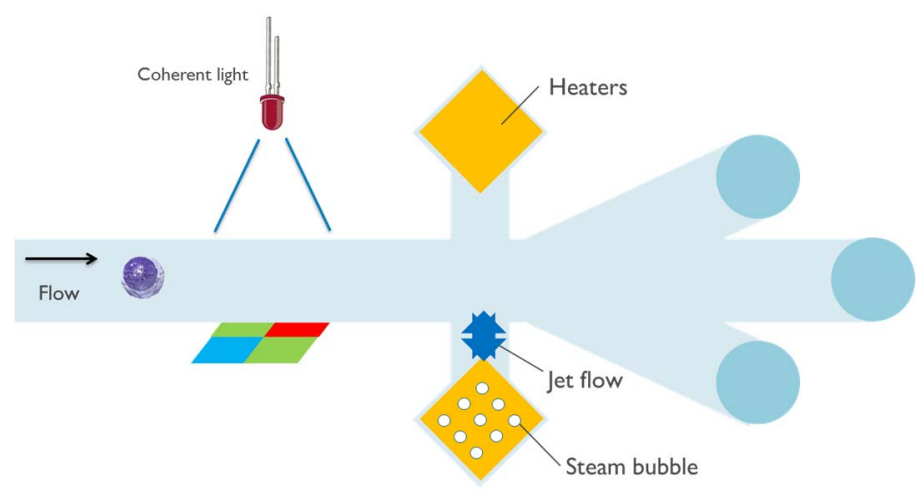

Fig. 1. Schematic diagram of basic principle of cell sorting based on jet flow

The device fabrication includes: processing of micro metal heaters, definition of polymer microfluidic channels and collective die-to-wafer bonding of glass substrate onto the polymer channels. In this paper we report on biocompatible polymer that is used for formation of microfluidics channels. This polymer is photo patternable and can be used as a bonding material to the glass die. This material has excellent resolution, has low curing temperature and has straight patterning profile essential for microfluidics channels. We will report on the automation, metrology and processing conditions for polymer adhesive material targeting a fabrication friendly process. The optimization of the bonding process by changing, time, temperature and pressure will be reported.

\section{Process flow}

The main step of the fabrication process are schematically shown in Fig 2. The processing starts with the formation of specifically designed tungsten microheaters (Fig. 2a) on to $200 \mathrm{~mm}$ Si wafer. These heater are then isolated with silicon nitride and silicon carbide (Fig. 2b). The electrical connection is made via Aluminium (Fig $2 \mathrm{c}$ ) and final isolation is done with silicon oxide. The silicon oxide above the heater and bond-pad is etched next. The use of silicon nitride and carbide stack help in reducing the thermal expansion mismatch and also help in creation of micro bubbles needed for the jet flow. Polymer is spin coated and patterned on the device wafer (Fig. 2d), followed by the collective bonding of upto 20 glass die onto the 
silicon wafer (Fig 2e). The glass die has pre-punched holes and used for bringing in and out the liquid sample. Important consideration during the development of the process was the scalability of the process for lower critical dimensions and compatibility of the process to a standard CMOS process.
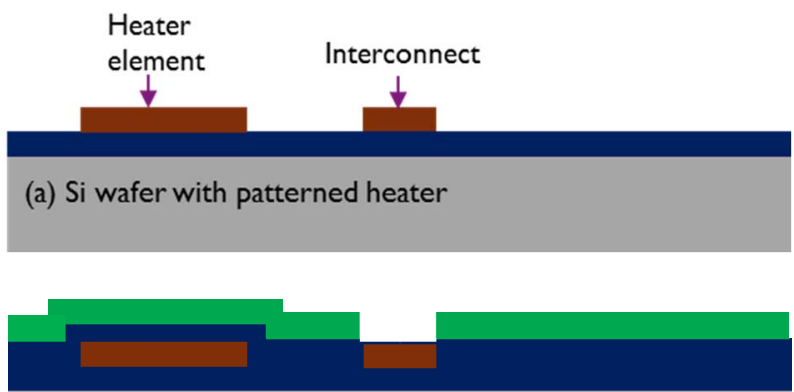

(b) $\mathrm{SiN} / \mathrm{SiC}$ cap over the heater
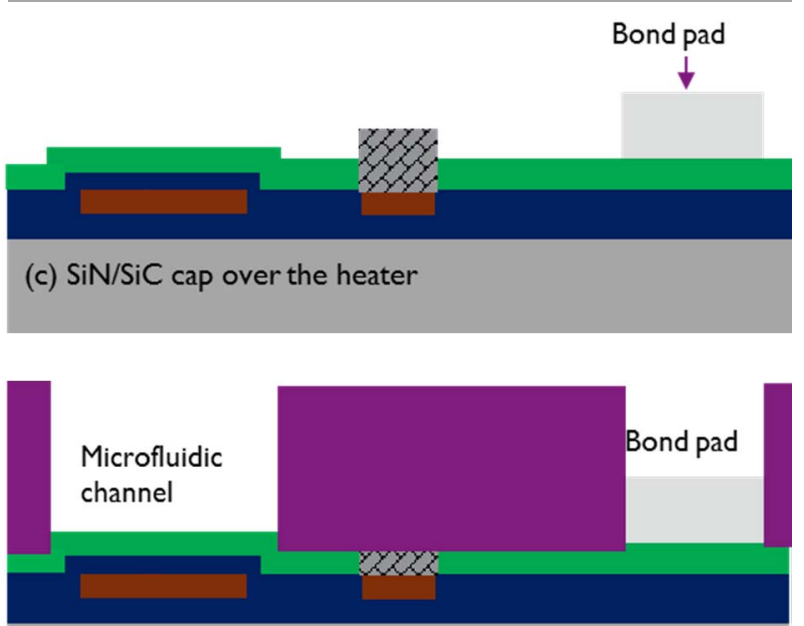

(d) Polymer patterning

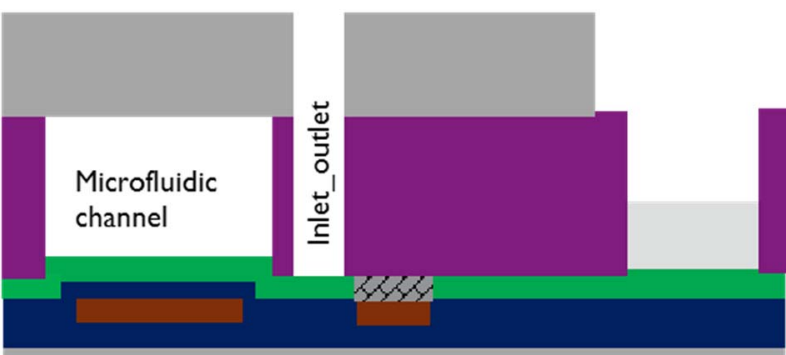

(e) Glass bonding

Fig. 2. Schematic process flow for the formation of the microfluidic device for cell sorting application

In the next section, a brief results from each of the step will be explained via a cross-section scanning electron microscopy (SEM). More explanation will be given to the characterization related to the polymer material. Initial work is done on 30 microns material and is reported in our initial work $[5,6]$. In this paper we look into the details for thicker polymer i.e. 60 microns and minimum $\mathrm{CD}$ is $30 \mu \mathrm{m}$. The thicker material is needed for cell analysis where the size of cell is larger than 30 microns. The polymer is negative tone and the exposure is done on 1X exposure tool with targeted mask which has a transmission factor greater than $90 \%$. We report on the process conditions and bonding condition optimization for the 60 microns polymer material.

\section{Results and discussion}

Fig. 3 shows the final results from the fabrication process till the deposition of polymeric layer. Tungsten microheater are etched with $\mathrm{SF}_{6}$ and $\mathrm{CH}_{3}$ chemistry to obtain very sharp profile as shown in Fig 3a. This is important because these corner acts as the nucleation site for the bubble generation. Fig $3 b$, shows the Tungsten heater protected with a layer of $\mathrm{SiN}$ and $\mathrm{SiC}$. Fig. $3 \mathrm{c}$ shows the addition of the Aluminum bond pad to the die. While Fig. $3 \mathrm{~d}$ shows the protective oxide that is removed from the heating element and bond pad opening.

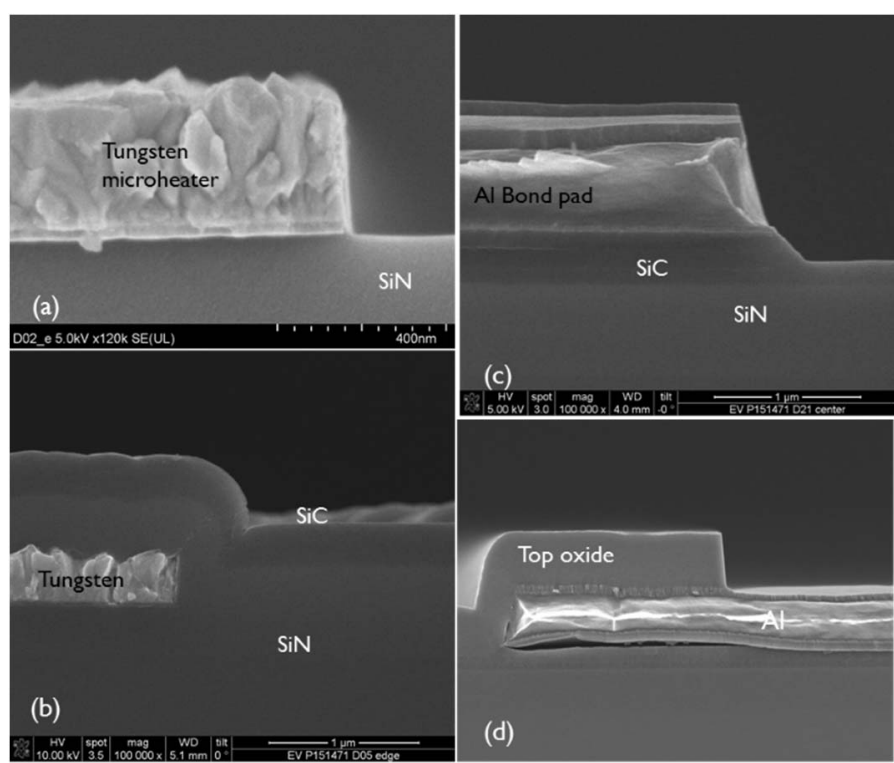

Fig. 3: Various SEM images of the device during the fabrication process.

\section{Characterization of polymer}

The first step was development of the spin curve to obtained the required thickness of the polymer material. Fig. 4 shows the spin curve indicating that 60 microns thickness is obtained with a double coating. During the first coating roughly 20 microns film thickness is obtained. While in the second coat for the same spin speed 40 microns film thickness is obtained. This is due to the fact that first layer is coated on a Silicon oxide wafer while the second layer interact differently with already coated polymer surface. 


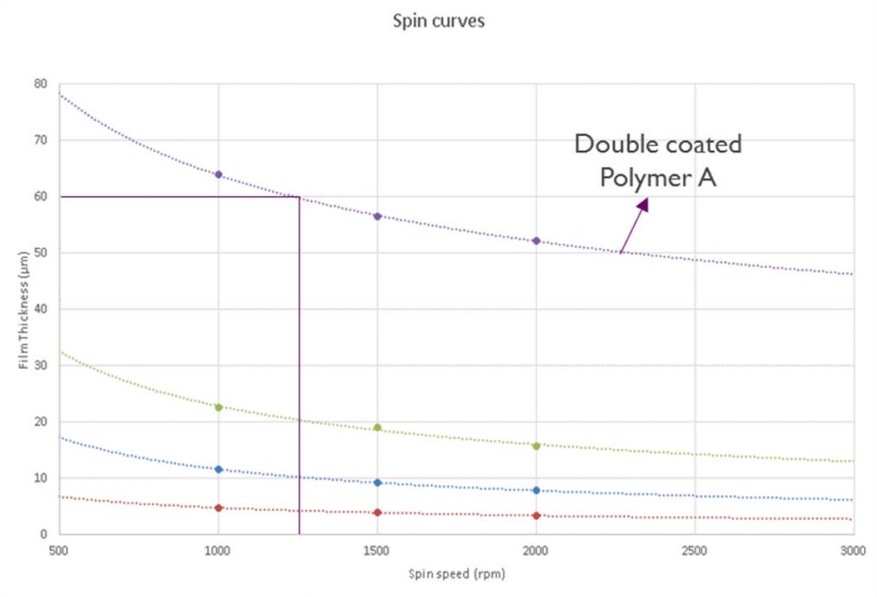

Fig. 4: Spin speed for dispensing 60 microns polymer

A design of experiment was done to determine the best conditions for the polymer. The main parameters analyzed were soft bake condition, focus and exposure energy.. A FEM showed that with i-line with focus $-25 \mu \mathrm{m}$ and dose of $2800 \mathrm{~mJ}$ provided the best result. Initially there was a difference in slope between the first and second layer of polymer giving a step profile. The different focus doesn't help avoiding the bad slope of the second layer. So soft bake and post exposure bake were analyzed. From the experiment it was concluded that with longer Soft bake gave a straighter profile while longer post exposure bake gave a more flat top surface that is good for bonding. The optimized results are obtained starting with dehydration bake of wafer @ 125dgC 180sec, manual dispense of material followed by the soft bake of $110^{\circ} \mathrm{C} 180 \mathrm{sec}$ coating for the first layer. The second layer is then coated with a softbake of $110^{\circ} \mathrm{C}$ for $300 \mathrm{sec}$ The development process used consisted of 3 cycles of the following sequence. $4.6 \mathrm{sec}$ dispense $+5 \mathrm{sec}+60 \mathrm{sec}$ puddle followed by 3 cycles of $60 \mathrm{sec}$ rinse with DI water. Fig. 5 shows the overview of the polymer coated on wafer.
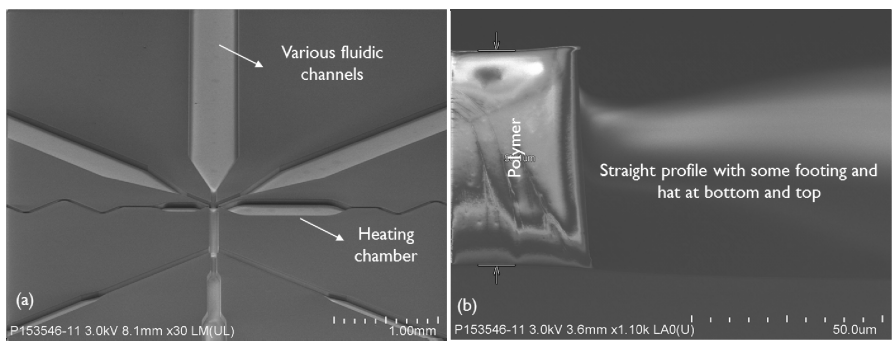

Fig. 5: Pattern definition of 60 microns polymer.

In the final aspect, we compared the thickness profiles of two materials and results are shown in Fig. 6. It shows the film uniformity across the wafer for 20 dies for the two thickness polymer. Fig. 6 a shows that 30 microns film is very uniform across the wafer and slight thickness is increased near the edge of the wafer typical to most spin coated material. However, for 60 microns polymer atypical profile is visible, with a central pattern visible where the thickness is slightly higher than average thickness as shown in Fig. 6b. This is linked to the interaction of polymer material with initial coated layer. The material is dispense by hand before the wafer is spun. This cause the polymer to stay slightly longer in this region thus giving this phenomenon. The results are tabulated with mean, max and TTV across the wafer for two thicknesses in table 1.
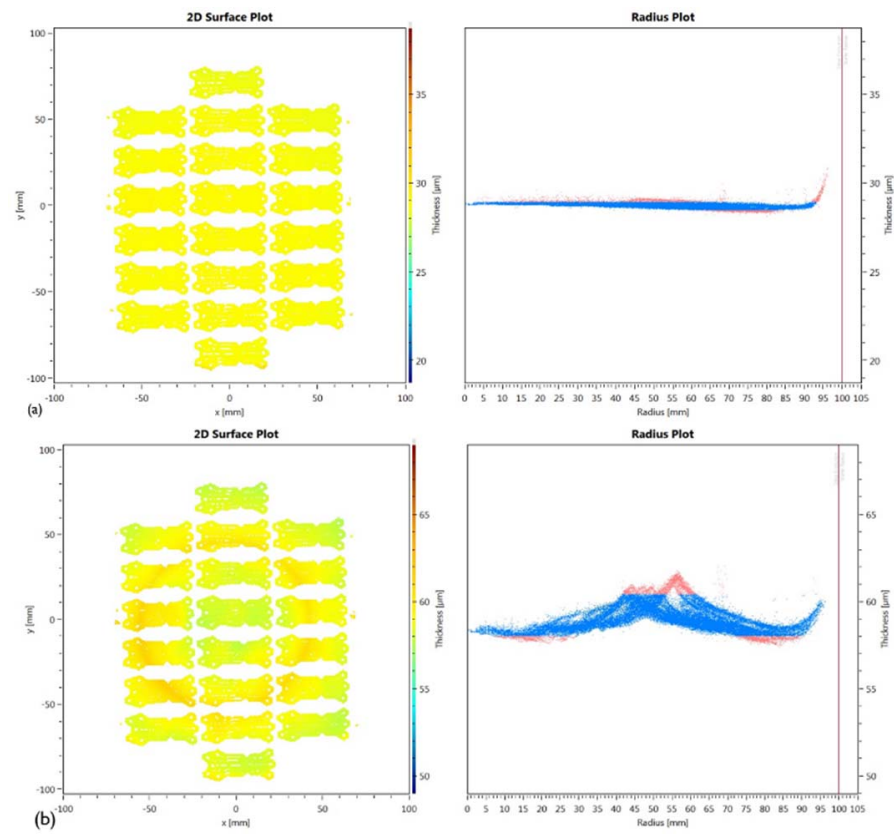

Fig. 5. Thickness analysis for two different thickness polymer

Table 1. Thickness uniformity for two different thickness polymers

\begin{tabular}{|l|r|r|r|r|}
\hline & $\begin{array}{c}\text { Mean } \\
\text { thickness }\end{array}$ & $\begin{array}{c}\text { Min } \\
\text { Thickness }\end{array}$ & $\begin{array}{c}\text { Max } \\
\text { thickness }\end{array}$ & TTV \\
\hline 30 mic PA & 28.72 & 28.12 & 30.83 & 2.70 \\
\hline 60 mic PA & 58.98 & 57.38 & 62.03 & 4.66 \\
\hline
\end{tabular}

\section{Characterization:}

\section{Leak test to check the bond strength}

To characterize the bonding, a fluidic test is carried out on dummy wafer with 30 microns polymer. The bonding is done in two step. In the first step, pre-punched glass die are aligned on to the matching pattern on the silicon wafer. After initial application of small force and temperature. In this way a total of 20 dies are populated on one wafer. In the second step, the final bonding is done in automated tool with application of force and temperature. The best bonding conditions were $300 \mathrm{~N}$ force per die at $200^{\circ} \mathrm{C}$ for 30 mintures. To check the quality, the test die is attached to external pumps and pressure is measured as function of flow rate. It shows that our devices can withstand a pressure in excess of 3 bar which is more than sufficient for most microfluidic applications such as cell sorting. The results are summarized in Fig. 6 


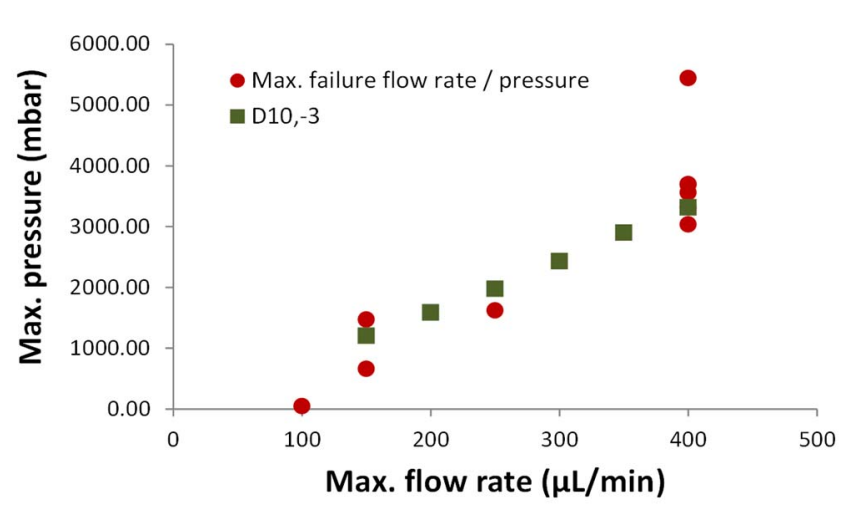

Fig. 6 Bonding quality check for max pressure as function of Max flow rate.

\section{Micro steam bubble generation}

In this stage, we characterize the formation of the micro bubble and how it can lead to jet flow that will help in sorting. A $0.16 \mathrm{~A}$ high current pulse was sent to the tungsten heater that is distributed to each hotspot for vapor nucleation. With this excitation condition, totally 256 bubbles emerged in the heater micro chamber and picture are taken by stroboscopic technique to determine the life time of the bubbles. Fig. 7 shows the different screen shots during the life time of the micro-bubbles. The steam bubbles have a minimum life time of less than $10 \mu \mathrm{s}$ and they can be classified as

$2 \mu$ s heating

$2 \mu \mathrm{s}$ (or longer) duration

$3 \mu \mathrm{s}$ cooling

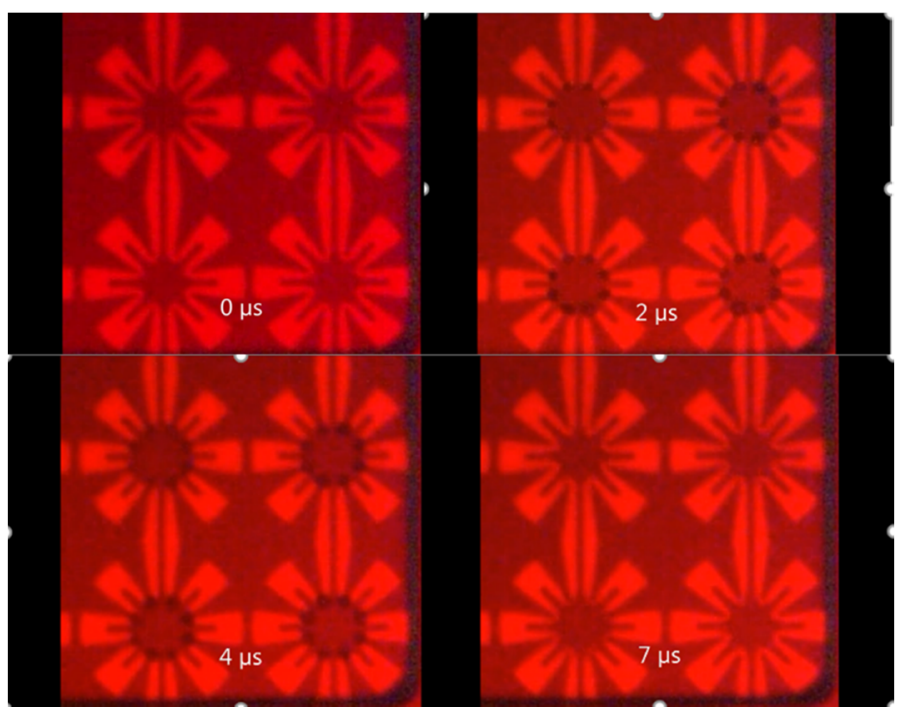

Fig. 7: Images at different time for the bubbles.

\section{Sorting efficiency}

The sorting efficiency of our device is tested using fluorescent beads. Fig. 8 shows screen shots from various stages of the sorting step. Fist at T0, the liquid is just passing through the microfluidic channel. After triggering of bubble jet flow, the flow is directed in one channel and remains in that direction till the bubble jet is stopped and after $0.1 \mathrm{~ms}$ the original state flow is maintained. Working with fluorescent beads Sort rate of 1,000 beads/s with sorter purity of up to $98 \%$ was achieved.

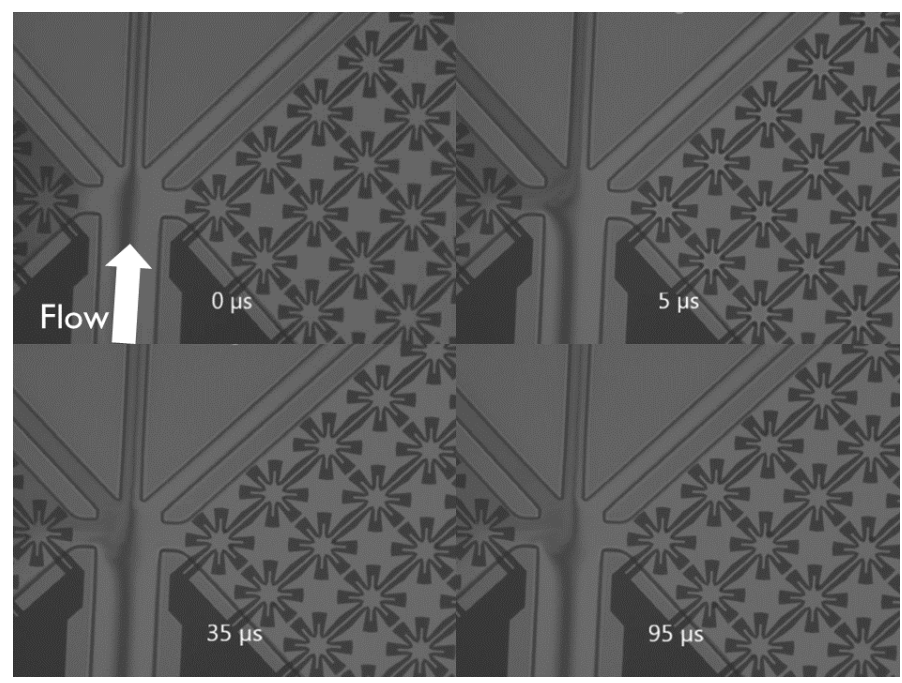

Fig. Screen shot of sorting principle

\section{Conclusions}

In this paper we report on a silicon fabrication process of a microfluidics device used for cell sorting application. The process uses a photopatternable polymer with excellent properties to combine fluidics and bonding application. The ability to process on wafer level distinguish this from other processes which can allow it to be a scalable technology. Details of the fabrication process along with results from the characterised sorting device were presented. Very high yield for sorting process is reported.

\section{Acknowledgments}

This project was supported by the European Research Council under the Consolidator Grant (SCALPEL; grant agreement no. 617312) The authors wish to Fab operation for fabrication of the devices.

\section{References}

1. C. W. Shields, C. D. Reyesab and G. P. Lopez, "Microfluidic cell sorting: a review of the advances in the separation of cells from debulking to rare cell isolation," Lab Chip, No. 15 (2015), pp. 1230-1249

2. Davey H. and Kell D., "Flow Cytometry and Cell Sorting Of Heterogeneous Microbial Populations: The Importance Of Single-Cell Analyses". Microbiological Reviews, Dec. 1996, p. 641-696

3. Picot j., Guerin C., Van Kim C., Boulanger C., "Flow cytometry: retrospective, fundamentals and recent instrumentation," Cytotechnology, Vol 64, Iss. 2, Mar 2012, pp. 109-130

4. A. Bhagat, H. Bow, H. Hou, S. Tan, J. Han, C. Lim, "Microfluidics for cell separation," Med Biol Eng Comput, vol. 48, pp.999-1014, 2010.

5. Liu C, de Wijs K, Vercruysse D, Majeed B., Dusa A, Miyazaki T., Deshpande P., and Lagae L., "A high speed microfluidic fluorescent cell sorter by micro thermal jet 
flow," CYTO 2014, Fort Lauderdale, USA (17-21 May 2014).

6. Majeed B., Liu C., Van Acker L., Daily R., Miyazaki T., Tezcan D., and Lagae L., "Fabrication of silicon based microfluidics device for cell sorting applications," In proc. IEEE 64 ${ }^{\text {th }}$ Electronic Components and Technology Conference ECTC. Orlando, USA. pp.165-169, 2014

7. Majeed, B.; Liu, C.; Sohn, E.; Van Acker, L.; de Wijs, K.; Sabuncuoglu Tezcan, D. and Lagae, L. "Silicon based cell sorting device: Fabrication, characterization and applications,"49 $9^{\text {th }}$ International Symposium on Microelectronics - IMAPS. Pasadena, CA, USA., 2016. 